\title{
Contrasting effects of fluoroquinolone antibiotics on the expression of the collagenases, matrix metalloproteinases (MMP)-1 and -13, in human tendon-derived cells
}

\author{
A. N. Corps, R. L. Harrall, V. A. Curry, B. L. Hazleman and G. P. Riley
}

Objectives. Fluoroquinolone antibiotics may cause tendon pain and rupture. We reported previously that the fluoroquinolone ciprofloxacin potentiated interleukin (IL)-1 $\beta$-stimulated expression of matrix metalloproteinases (MMP)-3 and MMP-1 in human tendon-derived cells. We have now tested additional fluoroquinolones and investigated whether they have a similar effect on expression of MMP-13.

Methods. Tendon cells were incubated for two periods of $48 \mathrm{~h}$ with or without fluoroquinolones and IL-1 $\beta$. Total ribonucleic acid (RNA) was assayed for MMP messenger RNA by relative quantitative reverse transcriptase polymerase chain reaction, with normalization for glyceraldehyde-3-phosphate dehydrogenase mRNA. Samples of supernatant medium were assayed for MMP output by activity assays.

Results. MMP-13 was expressed by tendon cells at lower levels than MMP-1, and was stimulated typically 10- to 100-fold by IL-1 $\beta$. Ciprofloxacin, norfloxacin and ofloxacin each reduced both basal and stimulated expression of MMP-13 mRNA. In contrast, ciprofloxacin and norfloxacin increased basal and IL-1 $\beta$-stimulated MMP-1 mRNA expression. Both the inhibition of MMP-13 and the potentiation of MMP-1 expression by fluoroquinolones were accompanied by corresponding changes in IL-1 $\beta$-stimulated MMP output. The non-fluorinated quinolone nalidixic acid had lesser or no effects.

Conclusions. Fluoroquinolones show contrasting effects on the expression of the two collagenases MMP-1 and MMP-13, indicating specific effects on MMP gene regulation.

KeY words: Collagenase, Fluoroquinolone, Interleukin, Matrix metalloproteinase, Tendon.

The fluoroquinolones are a widely used group of broad-spectrum antibiotics which target prokaryotic deoxyribonucleic acid (DNA) gyrase. Among other, generally mild, side-effects [1-4], fluoroquinolones may induce tendon pain and rupture in a small proportion (less than $1 \%$ ) of patients, an effect most frequently described for the Achilles tendon [5-7]. The factors predisposing individuals to suffer tendon damage remain undefined, although age, activity levels and ongoing treatment with corticosteroids have each been implicated [5-8]. Different clinical studies have reported a predominance of tendon problems with different members of the fluoroquinolone group (pefloxacin, ciprofloxacin, ofloxacin etc.), but the action on tendon appears to be a class effect [5-8].

Several studies suggest that alterations in the synthesis or breakdown of extracellular matrix (ECM) components may contribute to fluoroquinolone-induced tendon damage [9-12]. Treatment of rodents with pefloxacin produced a biphasic change in Achilles tendon proteoglycan synthesis, and oxidative damage to tendon collagen [11]. Both collagen irregularity and increased interfibrillar proteoglycan were described in a histopathological examination of a ciprofloxacin-associated Achilles tendinopathy [13]. In vitro studies indicated that increased expression of protease (caseinase) activity occurs in fluoroquinolone-treated cultured canine tenocytes [10], and we showed that ciprofloxacin potentiated the output of matrix metalloproteinase (MMP)-3 by interleukin (IL)-1 $\beta$-stimulated human tenocytes and increased the expression levels of both MMP-3 and collagenase 1 (MMP-1) messenger ribonucleic acid (mRNA) [12]. In the present study we have tested whether fluoroquinolones also affect the expression of a second IL- $1 \beta$-stimulated collagenase, MMP-13.

\section{Materials and methods}

\section{Materials}

Ciprofloxacin was obtained from ICN (Basingstoke, UK). Norfloxacin, ofloxacin, nalidixic acid and TRI-Reagent were from Sigma (Poole, UK). Fluoroquinolones were freshly dissolved at $10 \mathrm{mg} / \mathrm{ml}$ in $0.1 \mathrm{M} \mathrm{HCl}$, while nalidixic acid was dissolved at $10 \mathrm{mg} / \mathrm{ml}$ in $0.1 \mathrm{M} \mathrm{NaOH}$. Interleukin- $\beta$ was a gift from Glaxo Wellcome (Stevenage, UK). Dulbecco's Modified Eagle Medium (DMEM), fetal calf serum (FCS), antibiotics and oligonucleotide primers for reverse transcriptase polymerase chain reaction (RT-RCR) were obtained from Invitrogen (Paisley, UK). One-Step RT-PCR reagents and fluorescein (FAM)-labelled oligonucleotide probes were obtained from Applied Biosystems (Warrington, UK).

Achilles tendon specimens were obtained from tissue discarded during surgery, with approval by the Cambridge Local Research Ethics Committee and written consent from informed patients.

Rheumatology Research Unit, Box 194, Addenbrooke's Hospital, Hills Road, Cambridge CB2 2QQ, UK.

Submitted 1 May 2005; revised version accepted 1 August 2005.

Correspondence to: A. N. Corps, Rheumatology Research Unit, Box 194, Addenbrooke's Hospital, Hills Road, Cambridge CB2 2QQ, UK. E-mail: anc@mole.bio.cam.ac.uk 


\section{Cell isolation and incubation}

The isolation of tendon cells by outgrowth from tendon explants was described previously [12]. Cells (at passages between 4 and 10) were seeded at $10^{5}$ cells/well in six-well plates and were incubated for 3 days before the experiment. Cells were rinsed with $2 \mathrm{ml}$ of serum-free DMEM containing insulin, transferrin and selenium (ITS), and were given $2 \mathrm{ml}$ of the same medium containing fluoroquinolones $(50 \mu \mathrm{g} / \mathrm{ml})$ as required; control cells received equivalent additions of $0.1 \mathrm{M} \mathrm{HCl}$ or $\mathrm{NaOH}(\leq 1 \% \mathrm{v} / \mathrm{v}$, which did not significantly alter the $\mathrm{pH}$ of the medium). After $48 \mathrm{~h}$ the cells were rinsed and were given fresh medium with the same fluoroquinolone or control addition as in the pre-treatment, each with or without IL- $1 \beta(1 \mathrm{ng} / \mathrm{ml})$. After further incubation for $48 \mathrm{~h}$ the supernatant medium was removed and stored at $-20^{\circ} \mathrm{C}$, and the cells were rinsed with balanced salts solution and solubilized in TRI-Reagent ( $1 \mathrm{ml} /$ well).

This experimental design $(48 \mathrm{~h}$ pre-incubation with fluoroquinolones, followed by $48 \mathrm{~h}$ incubation with or without IL- $1 \beta$ in the continuing presence of fluoroquinolones) was adopted for consistency with our previous work showing potentiation of MMP-3 and MMP-1 expression [12]. We have found that a reduced, more variable, potentiation was obtained if the pre-incubation time was reduced and ciprofloxacin was added only at the same time as IL- $1 \beta$. Similarly, although the effects of fluoroquinolones described below were evident by $6 \mathrm{~h}$ of incubation with IL- $1 \beta$ in some experiments and by $24 \mathrm{~h}$ in each experiment, we have reported the data obtained after $48 \mathrm{~h}$ incubation for consistency.

\section{RNA isolation and analysis by RT-PCR}

Ribonucleic acid was isolated from the TRI-Reagent extracts by phenol-chloroform separation followed by precipitation with isopropanol and ethanol. The RNA was stored as aliquots at $-70^{\circ} \mathrm{C}$, and was assayed using One-Step RT-PCR in a GeneAmp 5700 (Applied Biosystems). All samples from a single experiment were run on the same plate, using either two or three replicate determinations per sample. The values obtained for MMP mRNA expression were corrected for glyceraldehyde-3phosphate dehydrogenase (GAPDH) mRNA expression in the same sample.

The primers and probes for GAPDH, MMP-1 and MMP-13 have been described previously $[14,15]$. Standard curves were run in each assay, using freshly diluted aliquots of pooled tendon cell RNA. For each target this produced a linear plot of threshold cycle $(\mathrm{Ct})$ against $\log$ (dilution), whose slope was within $10 \%$ of the expected value, indicating a similar, near-maximum efficiency. A single product of the appropriate size was produced, and no signal was produced if either the RNA or the reverse transcriptase step was omitted.

\section{Assays of MMP-1 and MMP-13 activity}

Serum-free culture supernatants were assayed for MMP-1 and MMP-13 using specific Fluorokine E fluorimetric assays as described by the supplier (R\&D Systems, Abingdon, UK). Samples with high MMP-1 content were diluted with assay diluent if required. Samples for MMP-13 assay were concentrated 5-fold using Centricon-10 concentrators (Millipore, Watford, UK). All samples were treated with 4-aminophenylmercuric acetate (APMA) to activate pro-enzyme prior to the assay, so that the total (latent and active) enzyme could be detected. Without APMA very little activity was recorded, consistent with our demonstration that most of the MMP-1 secreted by these cells was in the pro-enzyme form [12].

\section{Presentation of data}

The results presented are from five experiments performed separately with cells derived from five separate donors, in serumfree medium as described above. Similar results were obtained in parallel incubations in medium containing $10 \%$ FCS (data not shown), and aspects of the data for ciprofloxacin have been confirmed in at least six additional experiments. For data showing the effects of fluoroquinolones on basal MMP mRNA expression, the expression in control cells in each experiment was defined as $100 \%$. For data showing the effects of fluoroquinolones on IL- $1 \beta$-stimulated MMP mRNA expression, the expression in cells treated with IL-1 $\beta$ alone (after a control pre-incubation) was defined as $100 \%$, consistent with our previous report [12]. Significant differences from the $100 \%$ value were assessed using the $95 \%$ and $99 \%$ confidence intervals of the treated samples, and are presented in the figures as $P<0.05\left(^{*}\right)$ and $P<0.01$ (**).

\section{Results}

Tendon cells expressed mRNA encoding the collagenases MMP-1 and MMP-13. Basal expression of MMP-13 mRNA was at least 100-fold lower than that of MMP-1, as estimated from the $\mathrm{Ct}$ values in the optimized RT-PCR assays. Incubation of the cells with each of the fluoroquinolones and nalidixic acid reduced the basal expression of MMP-13 mRNA (Fig. 1A). Ciprofloxacin had the most consistent effects, and reduced MMP-13 mRNA expression in five out of six further experiments in addition to those shown in Fig. 1A. Interleukin- $1 \beta$ stimulated MMP-13 mRNA expression, typically 10 - to 100 -fold. Each of the fluoroquinolones also caused a significant reduction in MMP-13 mRNA expression in IL-1 $\beta$-stimulated cells (Fig. 1C), although it should be noted that IL- $1 \beta$ retained the ability to stimulate the expression of MMP-13 mRNA in the presence of fluoroquinolones. Nalidixic acid had no consistent effect on IL- $1 \beta$-stimulated MMP-13 mRNA expression (Fig. 1C).

Interleukin-1 $\beta$ also stimulated MMP-1 mRNA expression, typically 100 -fold. In marked contrast to their effects on MMP-13 mRNA, both ciprofloxacin and norfloxacin enhanced both basal and IL- $1 \beta$-stimulated expression of MMP- 1 mRNA, while ofloxacin and nalidixic acid had lesser or no effect (Figs 1B, 1D). This was observed both in serum-free medium (Fig. 1) and in medium containing $10 \% \mathrm{FCS}$; in the latter, ofloxacin had a smaller effect than ciprofloxacin or norfloxacin on basal MMP-1 expression, whereas ciprofloxacin had a greater effect than norfloxacin or ofloxacin on IL- $1 \beta$-stimulated MMP-1 expression $(P<0.05$ by Wilcoxon matched pairs test; $n=7$; data not shown).

The contrasting effects of fluoroquinolones on MMP-1 and MMP-13 mRNA expression were generally reflected in MMP output, measured by activity assays on the culture supernatants (Fig. 2). Interleukin-1 $\beta$-stimulated output of MMP-13 protein could be detected only after concentrating the culture supernatants, and corresponded to between 20 and $200 \mathrm{pg} / \mathrm{ml}$ over $48 \mathrm{~h}$ of stimulation by IL- $1 \beta$, compared with typically $40 \mathrm{ng} / \mathrm{ml}$ MMP-1 in the same samples. Ciprofloxacin and norfloxacin reduced MMP-13 output (Fig. 2A) and increased MMP-1 output (Fig. 2B). Ofloxacin or nalidixic acid had no significant effects (Fig. 2). We reported previously that ciprofloxacin did not consistently potentiate IL- $1 \beta$-stimulated MMP-1 output when assayed by western blotting [12]. However, such a potentiation of MMP-1 output by ciprofloxacin has been observed in the majority of subsequent experiments, although (as in the earlier work [12]) it has been smaller than the potentiation of MMP-3 expression in the same experiments. Thus, western blots of MMP-1 output in the present experiments showed a small potentiation of MMP-1 output by ciprofloxacin and norfloxacin that was consistent with the MMP-1 activity reported in Fig. 2B (V. A. Curry and A. N. Corps, data not shown). 

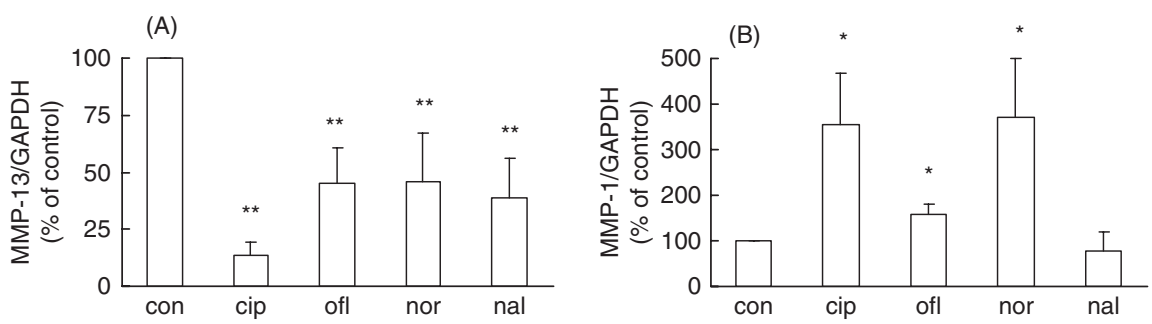

(C)

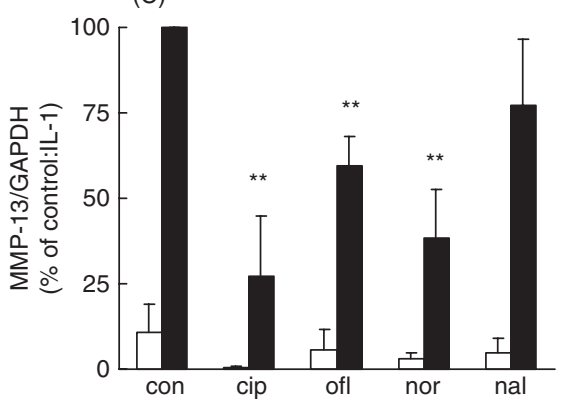

(D)

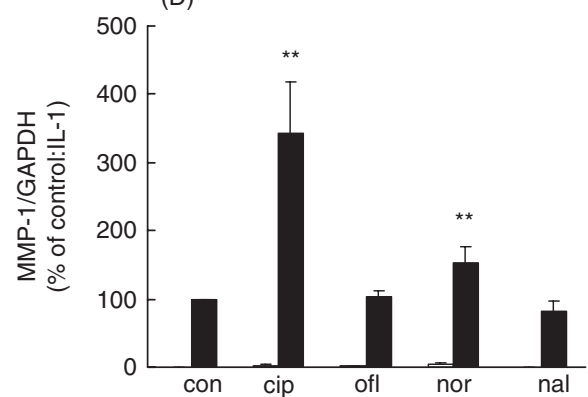

Fig. 1. Expression of MMP-13 and MMP-1 mRNA in human tendon-derived cells. Control cells (con) or cells pre-treated with $50 \mu \mathrm{g} / \mathrm{ml}$ ciprofloxacin (cip), ofloxacin (ofl), norfloxacin (nor) or nalidixic acid (nal), were then incubated for $48 \mathrm{~h}$ with the same fluoroquinolone or control addition as in the pre-treatment, each with or without IL-1 $\beta$ ( $1 \mathrm{ng} / \mathrm{ml}$ ). (A) MMP-13 and (B) MMP-1 mRNA in cells treated with fluoroquinolones alone were expressed relative to that in control unstimulated cells (defined as 100\%). (C) MMP-13 and (D) MMP-1 mRNA were expressed relative to that in IL-1 $\beta$-stimulated cells after control pre-treatment (defined as $100 \%$ ). Open columns, unstimulated cells; filled columns, IL-1 $\beta$-stimulated cells. Mean \pm S.E.M. from five experiments.

(A)

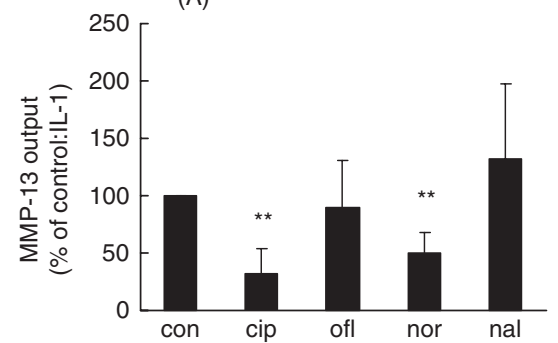

(B) $\quad$ **

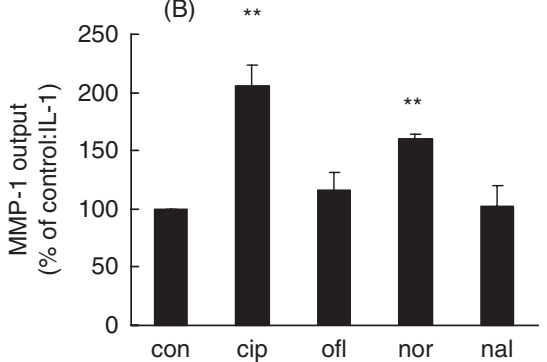

FIG. 2. Output of MMP-13 and MMP-1 from human tendonderived cells. Control cells (con) or cells pre-treated with $50 \mu \mathrm{g} / \mathrm{ml}$ ciprofloxacin (cip), ofloxacin (ofl), norfloxacin (nor) or nalidixic acid (nal) were then incubated for $48 \mathrm{~h}$ with the same fluoroquinolone or control addition as in the pre-treatment, each with or without IL-1 $\beta$ ( $1 \mathrm{ng} / \mathrm{ml})$. (A) MMP-13 and (B) MMP-1 activity in supernatant medium from IL- $1 \beta$-stimulated cells (filled columns) was expressed relative to that in medium from IL- $1 \beta$-stimulated cells after control pre-treatment (defined as $100 \%$ ). Mean \pm S.E.M. from three experiments. The values in the unstimulated cells (with or without fluoroquinolone treatment) ranged between undetectable and $5 \%$ of those in the IL- $1 \beta$-stimulated cells (data not shown).

\section{Discussion}

Tendon damage and rupture is a significant side-effect in a small proportion of patients treated with fluoroquinolones [5-8], and various studies have indicated that there are irregularities in ECM turnover in fluoroquinolone-treated patients and animal models $[9,11,13]$. Here we have found that the fluoroquinolones ciprofloxacin and norfloxacin increase and decrease, respectively, the expression of the collagenases MMP-1 and MMP-13. Expression of MMP-13 was markedly lower than that of MMP-1 in this culture system, but we cannot predict which of these contrasting effects of fluoroquinolones would be the more significant for collagenase expression or activity in the mechanical environment of the tendon in vivo.

Although we initially detected fluoroquinolone effects on MMP expression in the context of IL- $1 \beta$-stimulated expression of MMP-3 [12], several lines of evidence now indicate that their effects are independent of IL- $1 \beta$ receptors or IL- $1 \beta$ receptormediated signal transduction. First, fluoroquinolones affect basal expression of MMP-1 and MMP-3 (Fig. 1 and [12]), a result consistent with an earlier observation of increased protease activity from canine tendon cells [10]; they also have similar effects on the stimulation of these MMP induced by other growth factors (R. L. Harrall and A. N. Corps, unpublished data). Second, although they reduce the expression of MMP-13 they do not prevent stimulation by IL- $1 \beta$ above the reduced basal level (Figs 1 and 2). Third, ciprofloxacin did not affect early phosphorylation responses to IL-1 $\beta$ or its induction of cyclooxygenase-2 [16].

Further work will be required to define the molecular site(s) of action of the fluoroquinolones. Although fluoroquinolones decreased IL-1 $\beta$-stimulated output of prostaglandin (PG) $\mathrm{E}_{2}$, this did not explain the potentiation of MMP expression [16]. Recent studies using a rabbit tendon-derived cell line showed that ofloxacin was less effective than ciprofloxacin in generating reactive oxygen species (ROS) [17]. Those authors raised the possibility that ROS might be involved in the mechanism by which 
fluoroquinolones increase expression of MMP [17], and the similar relative potencies of ciprofloxacin and ofloxacin in both their study and ours are consistent with that hypothesis, which remains to be tested. The contrasting effects of fluoroquinolones on expression of MMP-1 and -13 implies that they may differentially affect one or more transcription factors which interact at the promoters of the MMP genes, for example AP1 or $\mathrm{NF} \kappa \mathrm{B}$ [18]. Using electrophoretic mobility shift assays, we have not detected effects of ciprofloxacin on either the basal or IL- $1 \beta$-stimulated activity of either of these factors in tendon cell extracts (A. N. Corps, unpublished data). An alternative approach would be to examine the effect of fluoroquinolones on the expression of transfected reporter plasmids driven by the MMP-1 and MMP-13 gene promoters, focusing on regions showing potential differences between the two promoters.

It is not clear how the order of fluoroquinolone potency that we have observed in vitro (ciprofloxacin and norfloxacin generally being more potent than ofloxacin) relates to clinical tendon problems. In a rat model of fluoroquinolone-induced tendon lesions, ofloxacin was more effective than ciprofloxacin and norfloxacin [19]. Clinical studies have often highlighted different individual fluoroquinolones [5-7], but, in a study presenting the adjusted odds ratios for Achilles tendon rupture in the elderly, the $95 \%$ confidence intervals for ofloxacin, ciprofloxacin and norfloxacin overlapped [8]. Given that the fluoroquinolones may affect the expression of different genes in opposite directions, as shown here, and that fluoroquinolones have been reported to affect the expression of other genes, such as those for cytokines [4], it is likely that altered MMP expression may be one of several factors contributing to tendon damage.

\begin{tabular}{|c|c|}
\hline & Key messages \\
\hline 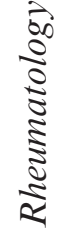 & $\begin{array}{l}\text { - Fluoroquinolone antibiotics show } \\
\text { differential effects on the expression of } \\
\text { collagenases by tendon cells. These } \\
\text { may contribute to the tendon problems } \\
\text { experienced by some fluoroquinolone- } \\
\text { treated patients. }\end{array}$ \\
\hline
\end{tabular}

\section{Acknowledgements}

This work was supported by the Arthritis Research Campaign (grants R0580, R0603 and 16125), the Cambridge Arthritis Research Endeavour, the Isaac Newton Trust and the Sybil Eastwood Trust.

The authors have declared no conflicts of interest.

\section{References}

1. Ball P, Mandell L, Niki Y, Tillotson G. Comparative tolerability of the newer fluoroquinolone antibacterials. Drug Saf 1999;21:407-21.

2. De Sarro A, De Sarro G. Adverse reactions to fluoroquinolones: an overview on mechanistic aspects. Curr Med Chem 2001;8:371-84.
3. Stahlmann R, Lode H. Fluoroquinolones in the elderly: safety considerations. Drugs Aging 2003;20:289-302.

4. Dalhoff A, Shalit I. Immunomodulatory effects of quinolones. Lancet Infect Dis 2003;3:359-71.

5. Pierfitte C, Royer RJ. Tendon disorders with fluoroquinolones. Therapie 1996;51:419-20.

6. van der Linden PD, van Puijenbroek EP, Feenstra $\mathrm{J}$ et al. Tendon disorders attributed to fluoroquinolones: a study on 42 spontaneous reports in the period 1988 to 1998. Arthritis Rheum 2001;45:235-9.

7. Khaliq Y, Zhanel GG. Fluoroquinolone-associated tendinopathy: a critical review of the literature. Clin Infect Dis 2003;36:1404-10.

8. van der Linden PD, Sturkenboom MC, Herings RM, Leufkens HM, Rowlands S, Stricker BH. Increased risk of Achilles tendon rupture with quinolone antibacterial use, especially in elderly patients taking oral corticosteroids. Arch Intern Med 2003;163:1801-7.

9. Burkhardt JE, Hill MA, Lamar CH, Smith GN Jr, Carlton WW. Effects of difloxacin on the metabolism of glycosaminoglycans and collagen in organ cultures of articular cartilage. Fundam Appl Toxicol 1993;20:257-63.

10. Williams RJ, Attia E, Wickiewicz TL, Hannafin JA. The effect of ciprofloxacin on tendon, paratenon, and capsular fibroblast metabolism. Am J Sports Med 2000;28:364-9.

11. Simonin M-A, Gegout-Pottie P, Minn A, Gillet P, Netter P, Terlain B. Pefloxacin-induced Achilles tendon toxicity in rodents: biochemical changes in proteoglycan synthesis and oxidative damage to collagen. Antimicrob Agents Chemother 2000;44:867-72.

12. Corps AN, Harrall RL, Curry VA, Fenwick SA, Hazleman BL, Riley GP. Ciprofloxacin enhances the stimulation of matrix metalloproteinase 3 expression by interleukin-1beta in human tendon-derived cells. A potential mechanism of fluoroquinoloneinduced tendinopathy. Arthritis Rheum 2002;46:3034-40.

13. Movin T, Gad A, Guntner P, Foldhazy Z, Rolf C. Pathology of the Achilles tendon in association with ciprofloxacin treatment. Foot Ankle Int 1997;18:297-9.

14. Ireland D, Harrall RL, Holloway G, Hackney R, Hazleman BL, Riley GP. Multiple changes in gene expression in chronic human Achilles tendinopathy. Matrix Biol 2001;20:159-69.

15. Corps AN, Curry VA, Buttle DJ, Hazleman BL, Riley GP. Inhibition of interleukin-1 beta-stimulated collagenase and stromelysin expression in human tendon fibroblasts by epigallocatechin gallate ester. Matrix Biol 2004;23:163-9.

16. Corps AN, Curry VA, Harrall RL, Dutt D, Hazleman BL, Riley GP. Ciprofloxacin reduces the stimulation of prostaglandin $\mathrm{E}_{2}$ output by interleukin-1b in human tendon-derived cells. Rheumatology 2003;42:1306-10.

17. Pouzaud F, Bernard-Beaubois K, Thevenin M, Warnet J-M, Hayem G, Rat P. In vitro discrimination of fluoroquinolones toxicity on tendon cells: involvement of oxidative stress. J Pharmacol Exp Ther 2004:308:394-402.

18. Vincenti MP, Brinckerhoff CE. Transcriptional regulation of collagenase (MMP-1, MMP-13) genes in arthritis: integration of complex signaling pathways for the recruitment of gene-specific transcription factors. Arthritis Res 2002;4:157-64.

19. Kashida Y, Kato M. Characterization of fluoroquinolone-induced Achilles tendon toxicity in rats: Comparison of toxicities of 10 fluoroquinolones and effects of anti-inflammatory compounds. Antimicrob Agents Chemother 1997;41:2389-93. 